\title{
REAL TIME IMAGING OF PROPAGATING HIGH FIELD DOMAINS IN SEMI-INSULATING GaAs
}

\author{
F. Piazza, P.C.M. Christianen and J.C. Maan \\ High Field Magnet Laboratory and Research Institute for Materials \\ University of Nijmegen \\ Toernooiveld, 6525 ED Nijmegen, The Netherlands
}

\begin{abstract}
With a newly developed technique, we measure voltage, electric field and charge distribution of high field domains in semi-insulating GaAs under high electric bias. Based on these new quantitative data resolved in time and space, which are synchronized with the current pulses, we confront the generally accepted model which explains the domain formation with field enhanced trapping from EL2.
\end{abstract}

PACS numbers: 72.20.Ht, 78.20.Jq, 07.60.-j, 78.47.+p

\section{Introduction}

In semi-insulating (SI) GaAs the application of a constant voltage of the order of $1 \mathrm{kV} / \mathrm{cm}$ gives rise to periodic current oscillations $(1-100 \mathrm{~Hz})$. This effect is caused by the $\mathrm{N}$-shaped negative differential resistance (NNDR) in the current versus electric field characteristic, where the uniform electric field distribution becomes unstable and splits into domains of high and low electric field. The presence of NNDR in SI GaAs is normally explained by field enhanced trapping from the midgap defect EL2. Two mechanisms have been proposed: trapping over a repulsive electrostatic barrier surrounding the trap [1] or scattering of hot electrons in a satellite $(L)$ valley in which the trapping is enhanced [2]. With a suitable electric field dependence of the trapping coefficient, numerical solutions of both models are able to reproduce high field domains traveling through the sample [3, 4]. Time dependent voltage, electric field and charge distributions have been calculated, but a quantitative experimental verification has not been possible up to recently [5]. For this reason we show here a space and time resolved measurement of traveling domains which provides for a severe test concerning the origin of slow domains. Moreover, this technique allows us to study the creation and annihilation of the domains, which is directly related to the current peaks. 


\section{Experiment}

The experimental setup is based on the longitudinal electrooptic effect of a $\mathrm{Bi}_{12} \mathrm{SiO}_{20}$ (BSO) crystal to transform a voltage distribution in a phase shift of light. The crystal has a transparent electrode on the front side and a dielectric mirror on the back side and is put with the back side on top of the sample. An expanded polarized laser beam enters the crystal through the transparent electrode and is reflected back by the mirror through an analyzer. Grounding the top electrode of the BSO, the phase shift induced by the voltage difference between the two sides of the crystal can be recorded as a light intensity distribution on a CCD camera. The laser source and the readout of the camera are synchronized with the current oscillations to resolve the measurement in time [5]. The sample is a $\langle 100\rangle$ LEC grown single crystal GaAs wafer: the mobility is $0.66 \mathrm{~m}^{2} /(\mathrm{V} \cdot \mathrm{s})$ and the electron density $3.1 \times 10^{12} \mathrm{~m}^{-3}$, with a thickness of $0.5 \mathrm{~mm}$ and having the NiAuGe contacts $8 \mathrm{~mm}$ apart. In Fig. 1 the voltage distributions on the sample are shown for three delay times with respect to the current pulse, at a bias of $1.6 \mathrm{kV} / \mathrm{cm}$. It is evident that a region of high field, high resistivity, which is formed at the cathode, moves through the sample towards the anode, without changing its shape.

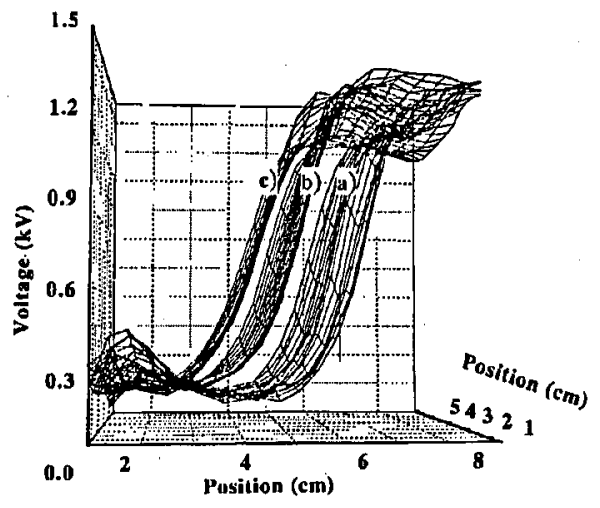

Fig. 1. Voltage distribution on a sample under a bias of $1.6 \mathrm{kV} / \mathrm{cm}$. Three different delays with respect to the current peak are presented: (a) $50 \mathrm{~ms}$, (b) $100 \mathrm{~ms}$, (c) $150 \mathrm{~ms}$.

The voltage, electric field and charge density distributions, obtained differentiating voltage along the axis of the sample, are shown in Fig. 2 as a function of the distance from the anode. Two important conclusions can be drawn from this picture:

- The peak electric field is much higher than $3 \mathrm{kV} / \mathrm{cm}$ necessary for intervalley scattering. This effect is important for the behavior of the trapping coefficient.

- The maximum charge density in the domain dipole corresponds to $2 \times 10^{17} \mathrm{~m}^{-3}$, which is much bigger than the equilibrium carrier density. Thus, the domains are mainly due to a fluctuation in space and time of the occupation number of the EL2 defects around the equilibrium value [6]. 


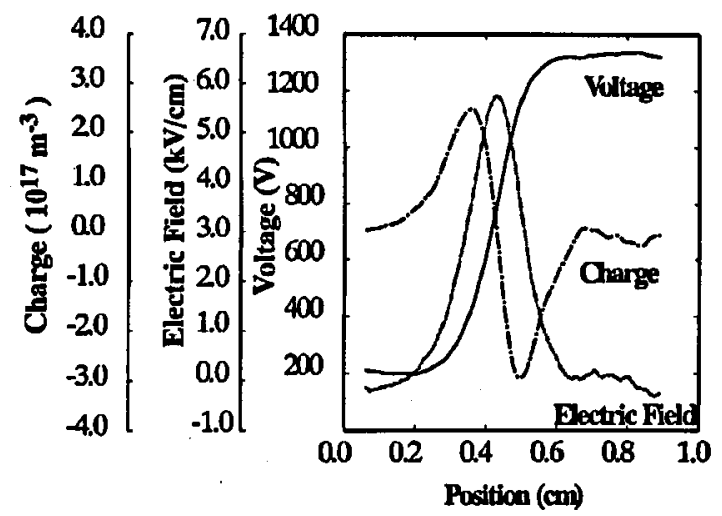

Fig. 2. Voltage, electric field and charge distribution in a domain. The bias voltage is $1.6 \mathrm{kV} / \mathrm{cm}$ and the time delay with respect to the current peak is $150 \mathrm{~ms}$. Voltage profile is directly measured while the electric field and the charge density are obtained via differentiation.
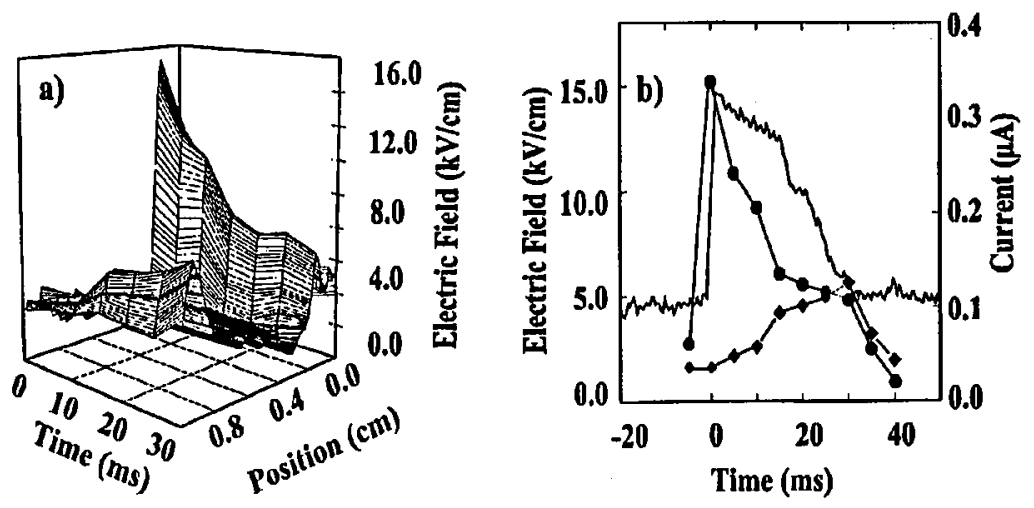

Fig. 3. Process of annihilation of one domain and creation of the next one in a sample under a bias of $1.6 \mathrm{kV} / \mathrm{cm}$. In part (a) the electric field along the axes of the sample is plotted as a function of the distance from the cathode and as a function of the time delay with respect to the rising edge of the current peak. In part (b) the current (line), the electric field peaks of the decreasing (circles) and of the growing (diamonds) domains are plotted as a function of time.

Figure 3 illustrates the correspondence between current signal and domain evolution under the same external conditions as Fig. 1. It can be seen that while the domain is propagating in the bulk the current level remains low. As soon as one domain reaches the anode there is a sudden increase in the peak electric field of the domain together with the raise of the current flow. The annihilation of the domain at the anode goes in parallel with the creation of the next one at the cathode. Once the electric field of the growing domain has reached the intensity 
of the traveling domains, it leaves the cathode even if the previous domain is not completely disappeared. This process is not clearly understood but we can state that:

- The peak in the current is not determined by the absence of a high resistivity zone in the sample as often assumed, instead at least one domain is always present.

- The peak in the current is not directly related to the transfer of charge from one domain to the next one. The current signal is low even if the domain at the anode is still decreasing. Moreover, the amount of charge transferred during the current peak is two orders of magnitude bigger than the charge inside the domain dipole.

- The current response seems to be dominated by the growing domain, while the effect of the disappearing one seems to be irrelevant.

In conclusion, we have measured quantitatively the time and space dependence of domains comparing data with existing models. New models and forthcoming experiments are necessary for improved understanding of this non-equilibrium transport phenomenon.

\section{References}

[1] H.K. Sacks, A.G. Milnes, Int. J. Electron. 28, 565 (1970).

[2] B.K. Ridley, J.J. Crisp, F. Shishiyony, J. Phys. C, Solid State Phys. 5, 187 (1972).

[3] S.P. McAlister, Z.-M. Li, Can. J. Phys. 69, 207 (1991).

[4] V.A. Samuilov, in: Nonlinear Dynamics of Pattern Formation in Semiconductor and Devices, Ed. F.-J. Niedernostheide, Springer, Berlin 1995, p. 220.

[5] B. Willing, J.C. Maan, Phys. Rev. B 49, 13995 (1994).

[6] E. Schöll, Physica B 135, 271 (1985). 